\title{
Sinopsis de los conocimientos sobre los Hongos Alucinogenos Mexicanos
}

\author{
Por Gastón Guzmán H. \\ Laboratorio de Botánica \\ Esc. Nal. de Ciencias Biológicas \\ I. P. N.
}

Debido a la posibilidad de que los hongos alucinógenos o psicotrópicos mexicanos ayuden a resolver problemas médicos, fundamentalmente relacionados con ciertas enfermerdades mentales, son hoy objeto de una intensa investigación por parte de diversos institutos en varios países.

Tales hongos fueron dados a conocer científicamente, de una manera más o menos firme, apenas en 1957 y 1958. Con anterioridad, en 1939 (27) y en 1949 (28) se habían tenido referencias aisladas de algunos de ellos, sin llegar a situarlos taxonómicamente.

Este trabajo es la condensación de las principales investigaciones realizadas hasta la fecha en Francia, Suiza, EE. UU. y México, y una síntesis de los resultados obtenidos por el autor, a través de sus trabajos emprendidos desde 1956. Lo que aquí se escribe ha sido ya expuesto, en tres conferencias dictadas por el autor, en el Seminario de Estudios Biológicos de la E.N.C.B. (agosto de 1957), en la Sociedad Botánica de México (abril de 1959) y en la Sociedad Mexicana de Microbiología (julio de 1959).

El autor agradece a los Srs. Dr. Rolf Singer de la Universidad de Tucumán, Argentina, Prof. Roger Heim del Museo Nacional de Historia Natural de París, Gordon Wasson de Nueva York y Prof. Teófilo Herrera y colaboradores del Instituto de Biología de la Universidad Nacional Autónoma de México, sus consejos y ayuda, facilitándole los trabajos que sobre este tema han publicado. Asimsimo, se le agradecen al Prof. Alfredo Barrera, valiosos consejos y sugestiones.

Numerosos escritos de la época de la Colonia, citan frecuentemente plantas que al ser ingeridas por los indígenas, les producían trastornos de tipo nervioso con percepción de visiones fantásticas: entre las más citadas están el "peyote" (Lophophora Williamsii), el "piule" u "ololiuqui" (Rivea corymbosa u otra Convolvulácea), el "toloache" (Datura stramonium y otras especies) y unos "honguillos" identificados con diversos nombres indígenas, según el autor de la cita.

Fray Bernardino de Sahagún (26) c:ta los hongos alucinantes con el nombre de "teonanácatl", palabra azteca que significa carne de los Dioses u hongo de los Dioses; según este escritor, los hongos - honguillos negros, como dice él- eran comidos por los indígenas en ceremonias especiales, causándoles lujuria y haciéndoles ver visiones. Francisco Hernández, en su

Guzmán-H. G. 1959. Sinopsis de los conocimientos sobre los hongos alucinógenos mexicanos. Boletín de la Sociedad Botánica de México 24: 14-34. 
"Historia de las Plantas de la Nueva España" (según Schultes, 27; Heim y Wasson, 21; Wasson, 37 y Aguirre-Beltrán, 2) cita a estos hongos con el nombre de "teyhuintle", denominación azteca que se traduce como embriagante. Motolinia (según Aguirre-Beltrán, 2; Guerra y Olivera, 4; Heim y Wasson, 21 y Wasson, 37) habla de los hongos alucinantes con el nombre de "teunanacatl". Jacinto de la Serna en su "Manual de Ministros de los Indios para el conocimiento de sus idolatrías y extirpación de ellas", según Wasson $(21,37)$, menciona en la región de Tenango del Valle (Méx.?) unos honguillos pequeños y dorados llamados "quautlannanacatl", vocablo que probablemente signifique hongo de los bosques (3), honguillos que eran comidos por los indios en ceremonias especiales. Fray Juan de Córdoba, en su "Vocabulario en Lengua Zapoteca", según Wasson (op. cit.), menciona unos hongos que emborrachan, mismos que identifica con los nombres de "péazoo", "peyazoo" y "nocuana peneeche", ésta última denominación significa honguillos arboricolas. Es interesante anotar que en la región de Huautla de Jiménez, Oax. (zona mazateca), existen 4 especies de hongos sagrados, con carácter estríctamente lignícola.

Los nombres citados por Sahagún, Hernández, Motolinia y Serna, no se usan en la actua idad entre los grupos indígenas adictos a los hongos, excepto por c'ertos herbolarios que usan las palabras "teyhuintle" o "teonanacate" (3).

La identificación botánica de tales hongos permaneció en total oscur'dad durante más de cuatro centurias. En 1915, Safford, según Schultes (27), los identifica con el "peyote" (Lophophora Williamsii), cactácea de propiedades alucinantes, afirmando dicho autor, que el "teonanácatl", no era más que el peyote seco, que a veces toma la forma caprichosa de un hongo.

En 1937, B. P. Reko, al hacer investigaciones etnológicas en México (25), colectó en la región de Huautla de Jiménez, Oax., unos hongos que identificó con el "teonanácatl". Schultes, en 1938 (27), siguiendo los pasos de Reko, colectó también en la misma región el supuesto "teonanácatl". El mater:al micológico de ambos investigadores, fué estudiado e identificado por D.H. Linder, de la Universidad de Harvard, dictaminando que la especie aludida era Panaeolus campanulatus $L$. var. sphinctrinus (Fr.) Bresadola, resultado que publicó Schu'tes, en 1939, en el boletín de la mencionada Universidad (27). Fig. 1.

De esta manera quedó asentado que el "teonanácatl” y los hongos afines a él, deberían pertenecer al género Panaeolus, principalmente a $P$. campanulatus var. sphinctrinus (el que ahora debe llamarse $P$. sphintrinus (Fr.) Quél.), así como a $P$. campanulatus y a $P$. papilonaceus. De estas tres especies, que son cosmopilitas, existía el antedecente de que en otras partes del mundo, sobre todo en Inglaterra y EE.UU., producían frecuentemente intoxicaciones de tipo nervioso con producción de alucionaciones $(23,24)$. 


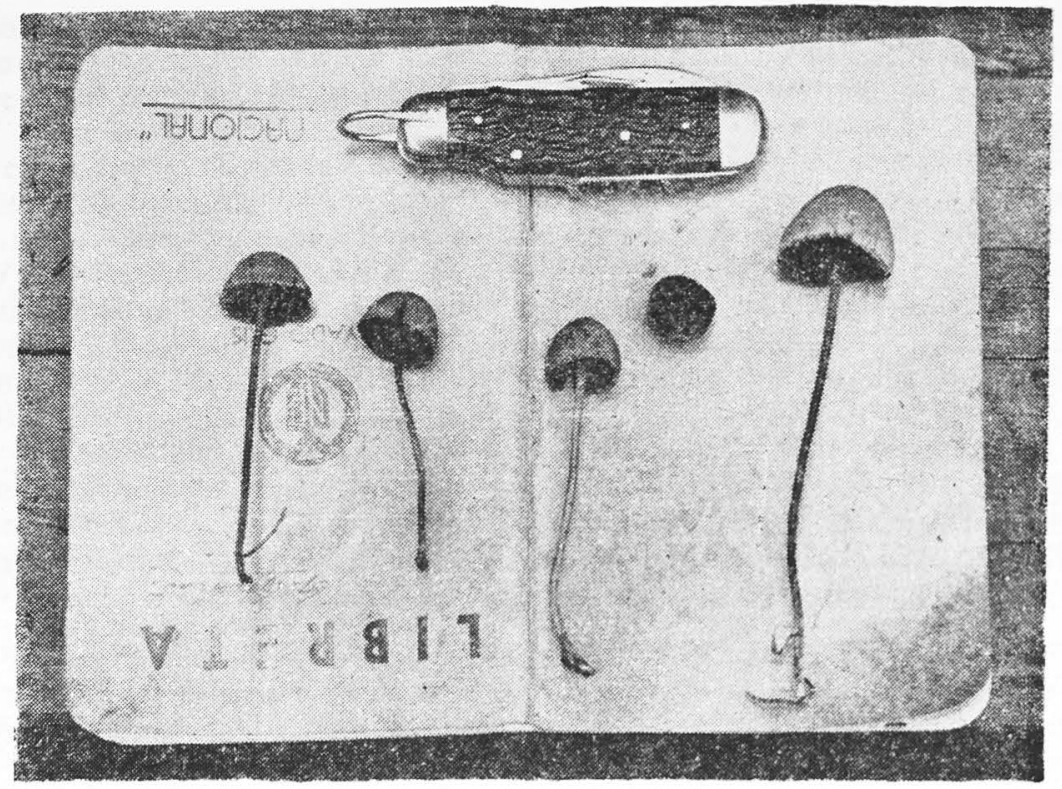

Paneolus sphinctrinus (Fr.) Quél el falso "Teonanácatl" de los aztecas. Hongo alucinógeno no usado por los indígenas.

Singer, en 1946, al estudiar el material colectado por Reko y Schultes, depositado en el Herbario de la Universidad de Harvard, descubrió que uno de los paquetes de la colecta, no correspondía a ningún Panaeolus, sino a Psilocybe cubensis (Earle) Sing. =Stropharia caerulescens (Pat.) Sing., Stropharia cubensis Earle) (28, 29, 30) anotando en 1949 (28) que Pitnaeolus sphinctrinus, $P$, papilonaceus y Psilocybe cubensis, presentaban propiedades alucinógenas a pequeñas dosis. Fig. 2.

La identificación del "teonanácatl" con Panaeolus campanulatus var. sphinctrinus se popularizó en varios círculos científicos y de esta manera se tuvo, hasta 1956, como el hongo alucinógeno mexicano más importante (4, 24, 36). En 1953, llegaron a México los esposos Wasson, que siguiendo sus aficiones etnomicológicas, se dedicaron a estudiar el "teonanácatl", adentrándose en todas las pob'aciones del país en donde según los cronistas y etnólogos, los indígenas comían hongos sagrados. El material micológico colectado por los Wasson, fué remitido a Roger Heim del Museo Nacional de Historia Natural de París, para su identificación. En 1956, Heim tuvo interés en visitar México, ya que la gran mayoría de los hongos que habían colectado los Wasson, eran especies no descritas científicamente; posteriormente dicho autor, de vuelta en París, emprendió un estudio minu- 


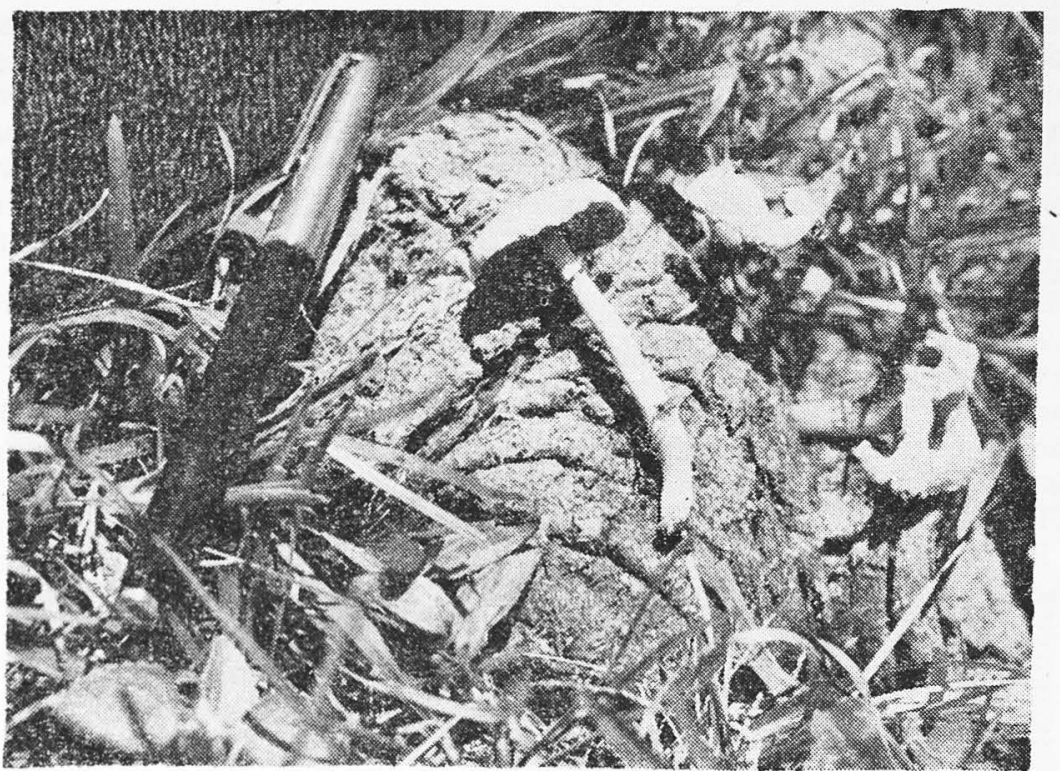

Psilocybe cubensis (Earle) Singer (Stropharia cubensis Earle, (según Heim) especie muy importante entre los mazatecos, común sobre el estiércol vacuno

cioso de todos los hongos alucinógenos mexicanos, logrando cultivar varias especies en el laboratorio y obtener material suficiente para hacer estudios químicos de los mismos. En sus múltiples trabajos, a partir de 1956, (no menos de veinte) ( 7 a 21 ) se han dado a conocer 13 especies de hongos alucinógenos mexicanos, de los cuales 9 especies y 2 variedades han resultado nuevas para la ciencia. En trabajo más recientemente publicado, hecho con la colaboración de Wasson y otros investigadores europeos (21), comprende una obra de 322 páginas, con 26 láminas a colores y numerosas figuras en el texto, en la que se expresan todas las investigaciones hasta ahora realizadas, principalmente en Europa, sobre los aspectos taxonómicos, químicos, fisiológicos, psíquicos y etnológicos, que sobre los hongos alucinógenos mexicanos se han llevado a cabo en aquel continente.

Independientemente de las investigaciones de Heim, Wasson y colaboradores, Rolf Singer, de la Universidad de Tucumán, Argentina, visitó México en 1957, para estudiar los hongos alucinógenos. El autor de estas líneas lo acompañó en sus viajes, por encontrarse en aquel tiempo ocupado también de la investigación de tales hongos. Singer, después de su estancia en México, pasó a Michigan, EE. UU., en donde en colaboración con A. H. Smith, estudió todo el material colectado, así como muestras que posterior- 
mente a su viaje,el autor le envió, dos de las cuales resultaron ser especies nuevas, más una nueva variedad $(34,35)$. Singer ha publicado hasta la fecha no menos de ocho trabajos referentes a los hongos alucinógenos mexicanos, entre ellos una monografía de la sección Caerulescentes Singer, 1948, del género Psilocybe (31) en que da a conocer 18 especies americanas de $P$ silocybe con posibles propiedades alucinógenas, de éstas, 9 son citadas de México, entre las cuales están 3 especies nuevas, así como una nueva variedad.

\section{Investigaciones Etnomicológicas}

La costumbre de ingerir hongos alucinógenos por parte de los indígenas, se conserva a pesar de que su práctica data desde tiempos precortesianos. Según las investigaciones de Wasson (21, 37, 37, 39), Reko (25), Schultes (27) y Johnson, Hoogshagen, Weitlaner, Stresser-Péan, Carrazco y Ravicz, entre otros (según Heim y Wasson, 21) y las investigaciones del autor, los indígenas que actualmente ingieren hongos narcóticos son:

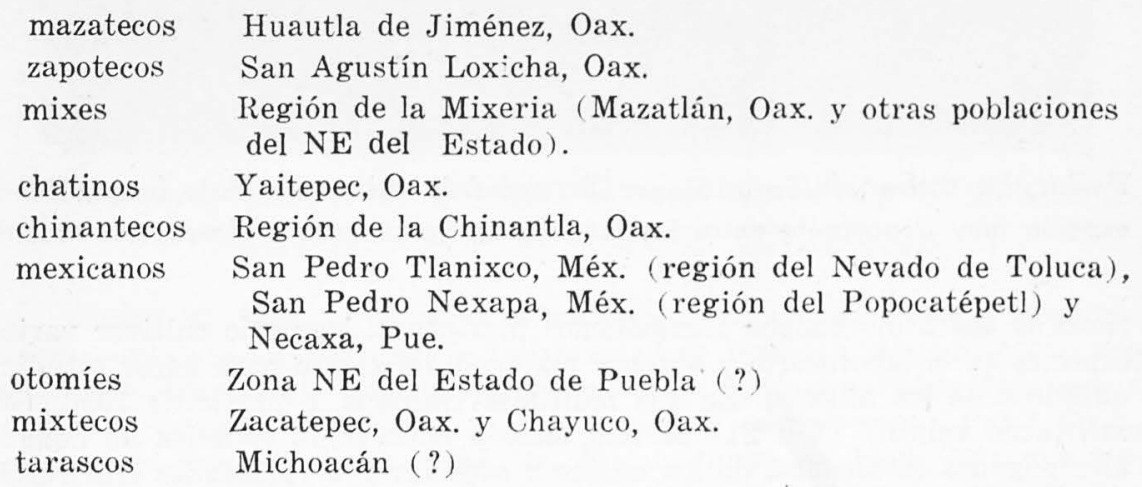

Los hongos en la mayoría de estos grupos etnológicos son motivo de adoración o respeto; únicamente son comidos en casos especiales, motivados por algún mal personal o de la comunidad. Siempre se ingieren en ceremonias nocturnas, presididas por algún curandero o por el jefe de la familia o de la comunidad, en donde los ritos paganos se han mezclado con los católicos. "Comemos los hongos para hablar con Dios y pedirle todo lo que deseamos", así se expresaron los mazatecos al ser interrogados por el autor, respecto a la causa que motivaba la ingestión de los hongos.

Casí siempre ingieren los hongos sin mezclarlos con ningún alimento; pero algunas veces, se toman con miel o chocolate para quitarles el sabor acre y astringente. Los mexicanos de San Pedro Tlanixco, Méx., acostum. bran ingerirlos mezclados con pulque. La "dosis" normal para los mazatecos es de 12 hongos (seis pares como dicen ellos, pues los hongos siempre 
se cuentan por pares, representando uno al marido y otro a la mujer). Los zapotecos y los mexicanos no usan en cambio una cantidad fija, variando ésta, según la edad y el estado de ánimo de la persona, pero en ningún caso la dosis llega a excederse de 25 hongos.

\section{Investigaciones Taxonómicas}

La taxonomía de los hongos alucinógenos ha sido muy discutida; los dos principales micólogos que se han dedicado a la clasificación e identificación de ellos son Roger Heim de Francia y Rolf Singer de Argentina y EE.UU., ambos por razones de criterio y método, no concuerdan en general en sus determinaciones, lo cuál ha dado lugar al establecimiento de sinomimias que hacen confuso su entendimiento.

La lista de las especies hasta ahora descritas de hongos mexicanos considerados como alucinógenos o psicotrópicos es la siguiente:

PSILOCYBE MEXICANA Heim, uno de los hongos alucinógenos subtropicales de mayor distribución en el país.

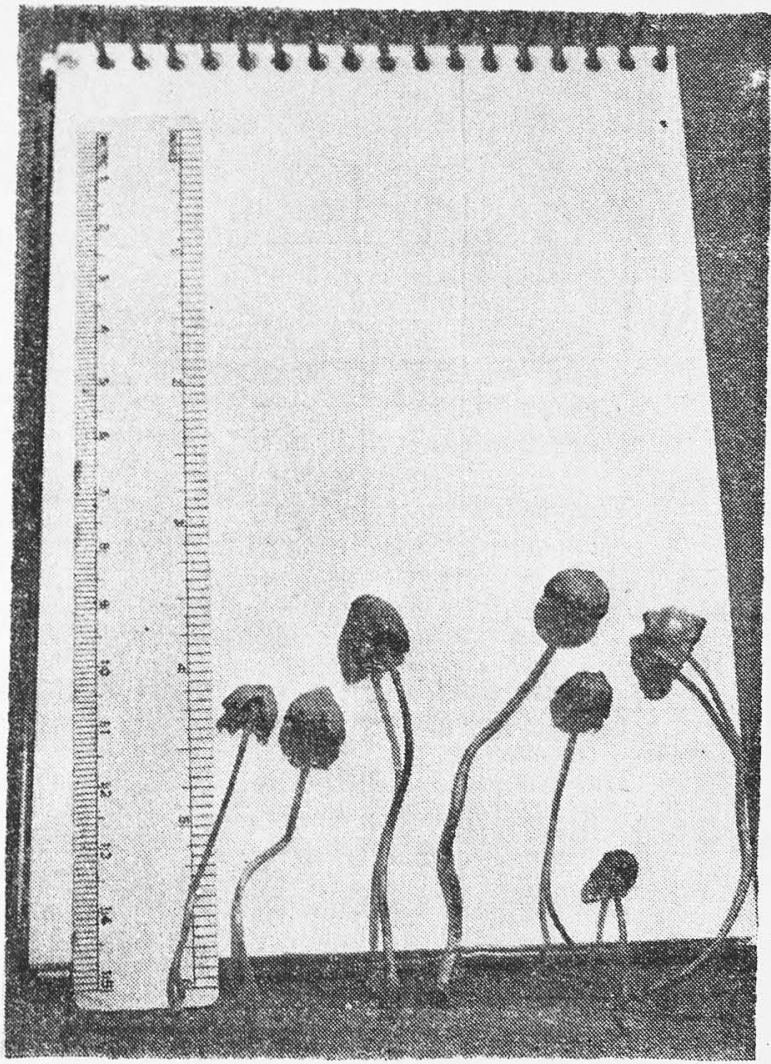


I. ESPECIES QUE INGIEREN LOS INDIGENAS

\section{A. VERDADERAMENTE PSICOTROPICAS}

Basidiomycetae, Agaricales, Strophariaceae

Psilocybe mexicana Heim, 1956 (ver Fig. 3)

Huautla de Jiménez, Oax. (mazatecos)

di-chi-to nizé (=hongo divino semejante a un pajarito)

di-nizé (=pajarito)

Angelitos

San Agustín Loxicha, Oax. (zapotecos)

piule de churis (=pequeño narcótico)

Yaitepec, Oax. (chatinos)

$c u i-y a-j o-t o-k i$ (=hongo santo de las praderas)

Mazatlán, Oax. (mixes)

pi-tpa (=parecido a un hilo)

Chinantla, Oax. (chinantecos)

a-mo-kia (=para adivinar) (?)

Necaxa, Pue. (mexicanos).

teotlaquilnanácatl

Ps. Zapotecorum Heim, 1956

San Agustín Loxicha, Oax. (zapotecos)

piule de barda (=narcótico de la corona de espinas de Cristo) razón giól

razón viejo

Yaitepec, Oax. (chatinos)

cui-ya-io-o-tnu (=gran hongo sagrado)

Huautla de Jiménez, Oax. (mazatecos)

di-nizé- ta-a-ya (=pajarito de monte) (?)

Ps.caerulescens Murr. var. Mazatecorum Heim, 1956 (ver Fig. 4)

(=Ps. caerulescens Murrill, 1923, según Singer y Smith)

Huautla de Jiménez, Oax. (mazatecos)

di-chi-tó ki-shó (=hongo divino de los derrumbes)

di-ki-shó (=derrumbito)

San Agustín Loxicha, Oax.

razón mbei (=hongo de la razón)

Ps. caerulescens Murr. var. nigripes Heim, 1956

(=Ps. caerulescens Murrill, según Singer) 


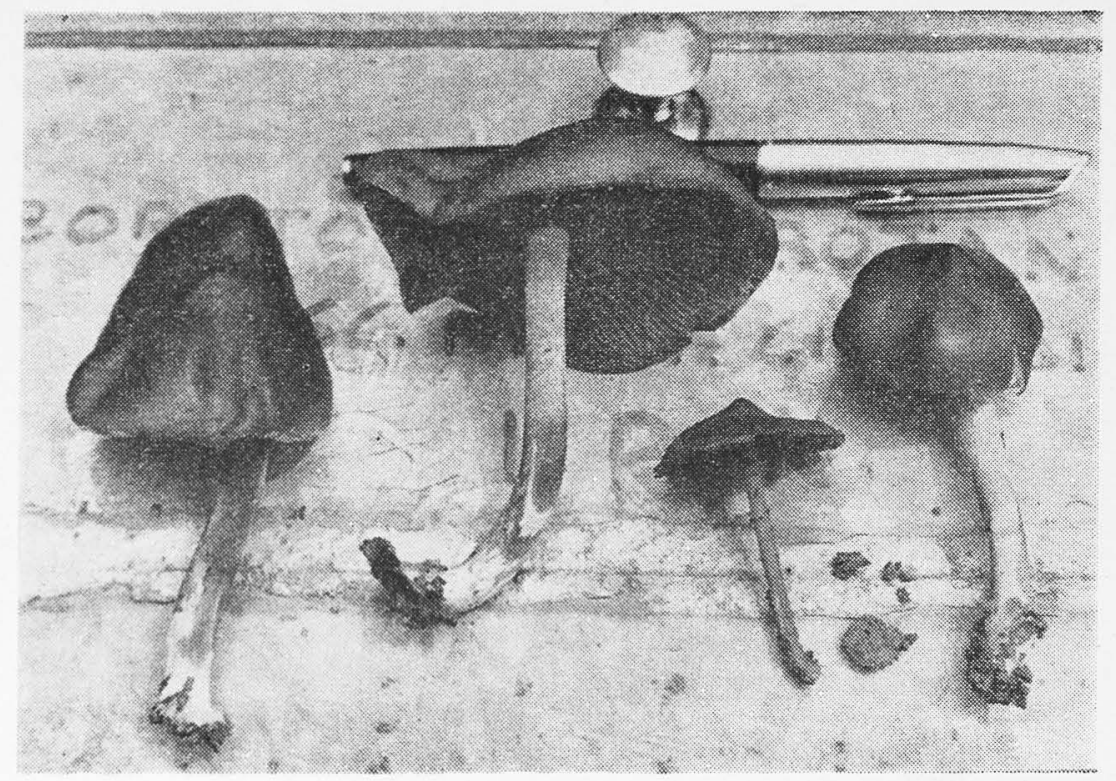

Psilosybe caerulescens Murr. var. Mazatecorum Heim Ps, caerulescens Murr. (según Singer), el "derrumbito" de los mazatecos; sumamente abundante en Huautla de Jiménez, Oax.

Yaitepec, Oax. (chatinos) cui-ya-jo-o-su (=hongo sagrado de gran poder)

Huautla de Jiménez, Oax. derrumbito negro

Ps. Aztecorum Heim, 1957

San Pedro Nexapa, Méx. (mexicanos)

apipiltzin (=niñito de las aguas)

dormilón (?)

Ps. muliercula Singer \& Smith, 1958

(=Ps. Wassonii Heim, 1958)

San Pedro Tlanixco, Méx. y Tenango del Valle, Méx. (mexicanos) siwatsitsintli (=mujercitas)

Ps. cubensis (Earle) Singer, 1951

(=Stropharia cubensis Earle, 1906, según Heim)

Huautla de Jiménez, Oax. (mazatecos)

di-shi-tjo-le-rra-ja (=hongo divino del estiércol del toro) 
Ps. yungensis Singer \& Smith, 1958

Huautla de Jiménez, Oax. (mazatecos)

di-nizé ta-a-ya (=pajarito de monte)

Ps. isauri Singer, 1958

Idem

Idem

Ps. caerulipes (Peck) Sacc. var. gastoni Singer, 1958

Idem

Idem

Ps. mixaeensis Heim, 1958

Coatlán, Oax. (mixes)

kong o kongk (=jefe)

Ps. cordispora Heim, 1956

Coatlán, Oax. (mixes)

atka-t (=alcalde)

Ps. Hoogshagenii Heim, 1958

Idem

Idem

B. DUDOSAMENTE PSICOTROPICOS

Ascomycetae, Hypocreales

Cordyceps capitata (Fr.) Link., 1833

San Pedro Tlanixco, Méx. y Tenango del Valle, Méx. (mexicanos) tlakatsitsintli (=hombrecitos)

C. ophioglosoides (Fr.) Link., 1833

Idem

Idem

Basidiomycetae, Bolbitiaceae

Conocybe siligenoides Heim, 1956

Huautla de Jiménez, Oax. (mazatecos)

di-nizé ta-a-ya (=pajarito de monte)

Basidiomycetae, Coprinaceae

Psathyrella sepulchralis Singer, Smith \& Guzmán

San Agustín Loxicha, Oax. (zapotecos)

piule de barda $\mathrm{A}=$ narcótico de la corona de espinas de Cristo) 
Panaeolus fimicola (Fr.) Guillet

Huautla de Jiménez, Oax. (mazatecos)

(no se ha citado nombre vulgar)

Basidiomycetae, Strophariaceae

Psilocybe acutissima Heim, 1958

Huautla de Jiménez, Oax. (mazatecos)

(no se ha citado nombre vulgar)

Basidiomycetae, Gasteromycetales

Dictyophora phalloides Desvaux

Quetzalapa, Oax. (chinantecos)

(no se ha citado nombre vulgar)

\section{FALSAMENTE PSICOTROPICOS}

Basidiomycetae, Polyporales, Cantharellaceae

Cantharellus flocosus Schaw. (= Nevrophyllum Heim Gomphus Singer.)

(confundido en la práctica con las especies de Cordyceps)

Basidiomycetae, Polyporales, Clavariaceae

Clavaria pistilaris $L$.

(Idem)

Basidiomycetae, Agaricales, Cortinariaceae

Cortinarius cortinaceus f. largusoides Henry

(confundido en la práctica con Psilocybe yungensis y afines)

Basidiomycetae, Agaricales, Rhodophyllaceae

Rhodophyllus $s p$.

(Idem)

Conocybe tenera (Schaeff. ex Fr.) Fayod ex aut. (confundido en la práctica con Psillocybe mexicana)

C. siliginea (Fr.) Kühner

(Idem)

Basid:omycetae, Agaricales, Strophariaceae

Stropharia phallaciosa Heim

(confundido en la práctica con Psilicybe Aztecorum)

Psilocybe aff. siccipes Karst.

(confundido en la práctica con $P s$. mexicana) 
II. ESPECIES QUE NO INGIEREN LOS INDIGENAS

A. VERDADERAMENTE PSICOTROPICAS

Basidiomycetae, Agaricales, Amanitaceae

Amanita muscaria (Fr. ex L.) Pers. ex Gray

Basidiomycetae, Agaricales, Strophariaceae

Psilocybe candidipes Sing. \& Smith

Basidiomycetae, Agaricales, Coprinaceae

Panaeolus sphinctrinus (Fr.) Quél.

B. DUDOSAMENTE PSICOTROPICOS

Basidiomycetae, Agaricales, Coprinaceae

Anellaria sepulchralis (Berk.) Br. Singer

Respecto de la lista de los hongos alucinógenos anteriormente enumerados, cabe hacer los siguientes comentarios:

1.-Según Heim, los hongos alucinógenos mexicanos están representados por Psilocybe, Stropharia y Conocybe. Según Singer, la única especie de Stropharia dentro de los hongos alucinógenos queda incluída en el género Psilocybe, además Conocybe siligenoides, según el aludido autor, es totalmente independiente de los hongos alucinógenos, de tal manera que éstos quedan solamente integrados por el género Psilocybe.

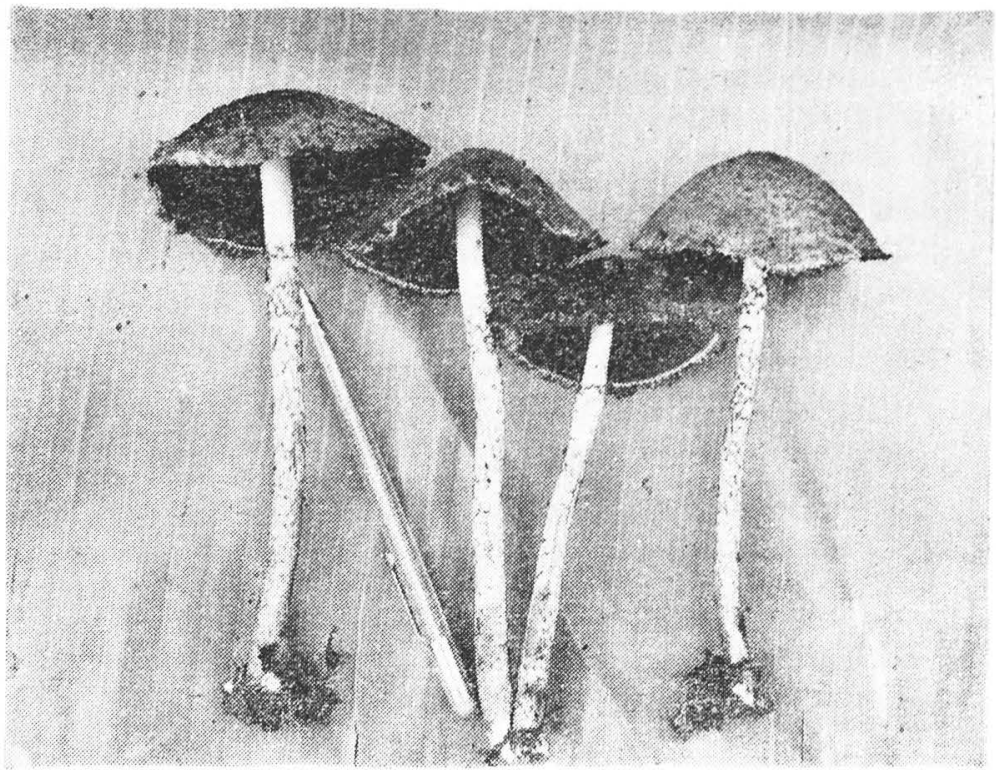

Psathyrella sepulchrais Singer, Smith \& Guzmán, especie que localizó el autor en el cementerio de San Agustín Loxicha, Oax.; los indigenas la confunden con Psiloscybe Zapotecorum Heim. 
2.-Todas las especies verdaderamente alucinógenas del género Psilocybe quedan incluídas según Singer, en la sección Caerulescentes del mismo género.

3.-Conocybe siligenoides es probable que los mazatecos lo confundan con Psilocybe yungensis, Ps. isauri y Ps. caerulipes var. gastoni. Psathyrella sepulchralis lo confunden los zapotecos con Psylocybe Zapotecorum. Fig. 5.

4.- - Singer y Smith sospechan que aparte de las especies de Psilocybe de la sección Caerulescentes, están también entre los hongos de acción neurotrópica, algunas especies que tienen la facultad de ponerse de color azul al contacto con el aire, tales como Copelandia cyanescens, $\mathrm{Pa}$ naeolus subalteatus y algunas especies de Pholiotina y Mycena.

5.-Dentro de la familia Russulaceae de los Agaricales, existe la Russula nordorbingi Singer, especie de propiedades psicotrópicas comprobadas, únicamente citada de Nueva Guinea $(32,29)$.

6.-El autor cuenta con dos especies del género Psilocybe, de propiedades alucinógenas, según los indígenas y cuya determinación no concuerda con las clasificaciones de Heim y de Singer. Ambas están en estudio.

7.-Heim y Cailleux obtuvieron de los cultivos de Psilocybe mexicana experimentados en París, una especie mutante (!), la cual han llamado Psilocybe sempervivae Heim et Calleux (18).

\section{Investigaciones Químicas y Fisiológicas}

Los trabajos químicos sobre los hongos alucinógenos mexicanos fueron iniciados en 1939 por Santesson en Estocolmo, Suecia, con material que Reko le envió desde México, colectado en Huautla de Jiménez, Oax. en 1937 ( según Reko, 25 ; Singer 30) Santesson en su trabajo: "Einige mexikanische Rauschdrogen" (Ark. Bot. 29a (12): 1-9, 1939, en Singer op. cit. afirma haber aislado el principio activo de tales hongos, una especie de glucósido que al ser administrado sobre ranas, produjo un efecto similar al de la morfina. La identiificación botánica del mater:al experimentado fué dada muy vagamente, sospechándose únicamente que se trataba de Panaeolus sp. (probablemente P. campanulatus var. sphintrinus) y Armillarea mellea. Según Singer, el material analizado por Santesson, fué una mezcla de Psilocybe mexicana (falsamente llamado Panaeolus sp.) y Ps. cubensis (falsamente identiifcado como Armillarea mellea).

No fué sino hasta 1958 cuando los Laboratorios Sandoz, de Basilea, Suiza, Hofmann y sus colaboradores (22), aislaron de una manera definitiva el principio activo de Psilocybe mexicana, un alcaloide al que han llamado psilocybina; se caracteriza éste por tener una estructura indólica 
con fósforo derivada de la triptamina. Fué aislada además, en pequeñas cantidades, una segunda sustancia de composición química semejante, caracterizada por no tener fósforo, y a la cual se le llamó psilocina.

Posteriormente, la psilocybina ha sido aislada de Ps. cubensis, Ps. Zapotecorum, Ps. caerulescens var. Mazatecorum, Ps. Aztecorum y "Ps. semperviva", e inclusive de Panaeolus sphinctrinus. El haber encontrado psilocybina en esta última especie, descarta la idea de que fuera exclusiva del género Psilocybe (de la sección Caerulescentes de Singer) y comprueba la sospecha de que Panaeolus produce efectos alucinógenos, tal como lo citan ciertas publicaciones sobre intoxicaciones de tipo nervioso, producidas con diversas especies del género, entre ellas $P$. sphinctrinus, $P$. campanulatus, $P$. papilonaceus, $P$. venenosus ( $=P$. subalteatus) y otros $(23,24,32)$. Tyler en mayo de 1958, según Heim y Wasson (21), puso de manifiesto la existencia de serotina en Panaeolus campanulatus; según Hofman y Heim (op. cit.) dicha especie no tiene psilocybina. Es probable que $P$. campanulatus, de no tener psilocybina, posea algún compuesto semejante con propiedades psicotrópicas, relacionado con la $s e$ rotina.*

En noviembre de 1958 fué dado a conocer por Hofmann y colaboradores, de los Laboratorios Zandoz, la síntesis de la psilocybina, a partir del 2-nitro-6-hidroxi-tolueno, descubrimiento que representa un gran adelanto para el conocimiento de la acción neurotrópica de los hongos alucinógenos en general.

La pasilocybina, lo mismo que la psilocina, no son las únicas sustancias responsables de la acción neurotrópica de tales hongos, debido a que Psilocybe yungensis, especie con propiedades alucinógenas comprobadas por Wasson (21), ha dado reacción negativa en cuanto a la presencia de tales sustancias, según Heim y Wasson (21).

Amanita muscaria es una especie alucinógena, común en varias regiones forestales del mundo; en México es exclusiva de los bosques de Pinus spp., a la vez que es totalmente ignorada por los indígenas adictos a los hongos alucinógenos, no así por los de una tribu de Kamchatka, en Siberia, que la comen de manera semejante como los psilocybes en México (24, 29, 38). Químicamente ha sido muy estudiada desde el siglo pasado y así se tenía como responsable de la acción tóxica del hongo a la muscarina, glucósido que modernamente se ha visto sólo existe en un $0.003 \%$ y que de ninguna manera es responsable de la acción alucinógena. En 1953 se aisló de Amanita muscaria la bufotenina (1), alcaloide afin a la psilocybina y a la serotina, primeramente descubierto, en 1920, de la piel de los sapos (Bufo sp.). Recientemente la bufotenina ha sido sintetizada en Michigan,

x La serotina (5-hidroxi-triptamina) es una hormona aislada en 1951, coún en la sangre de los mamiferos y en los cuales presenta una acción vasoconstrictora. 
EE.UU. y experimentada sobre el cuerpo humano a dosis variable; $10 \mathrm{mg}$. produjeron alucinaciones, hormigueo en el cuerpo y somnolencia.

La ingestión de los hongos alucinógenos del género Psilocybe de la sección Caerulescentes, se caracteriza por estimular el sistema nervioso, principalmente el neurovegetativo, provocando alucinaciones. Los síntomas en orden de aparición son: midriasis; relajamiento muscular; flacidez; dificultad para andar y hablar; dificultad para pensar y concentrarse en algo; emotividad excesiva; hilaridad; percepción de ilusiones y alucinaciones, primero de tipo cromático, después auditivas de ruidos y voces; estado de depresión y pérdida de las nociones del tiempo y del espacio. Lo interesante del caso es que la persona que ingiere hongos, no olvida su identidad y todo lo que siente y ve, lo relaciona con sí misma. La dosis normal que ingieren los indígenas varía entre 6 a 25 hongos, llegando a pesar cada hongo entre 0.75 gramos en fresco y 0.7-0.5 gramos en seco, aproximadamente, variando la dosis, según el estado de emotividad del individuo, la edad, el tamaño de los hongos, etc. La ingestión de tales hongos no forma hábito, ni tiene efectos acumulativos. Dosis grandes pueden provocar casos graves de locura e incluso la muerte, no así dosis pequeñas que apenas producen relajamiento muscular, somnolencia e hilaridad, únicamente.

En los citados Laboratorios Sandoz, de Basilea, Suiza, se han realizado estudios farmacológicos de la psilocybina, investigaciones que han demostrado que ésta es menos tóxica que la bufotenina. Dosis de psilocybina de $250 \mathrm{mg} . / \mathrm{Kg}$. en animales de laboratorio, empiezan apenas a provocar mortalidad, contra $25-30 \mathrm{mg} / \mathrm{kg}$. de bufotenina. Los síntomas que se producen en los animales de laboratorio, bajo la acción de la psilocybina, fueron: dilatación de la pupila, piloerección, taquicardia, elevación térmica, hiperglicemia, elevación de la presión sanguínea y contracción de la membrana nictitante, entre otros. La administración periódica de psilocybina, no mostró ningún síntoma de acostumbramiento hacia ella.

Todos los síntomas que produce la ingestión de los hongos psicotrópicos, ya sea silvestres o cultivados, han sido comprobados personalmente, por Wasson, Heim, Cailleux, Hofmann y otros investigadores (11, 21, 38). En México, los hongos cultivados en el Instituto de Biología, pertenecientes a Psilocybe cubensis (40), han sido administrados a estudiantes a manera de experimentación. Por otra parte, el autor, estando en la población de Huautla de Jiménez, Oax., el 7 de agosto de 1957, ingirió la dosis normal para los mazatecos de "hongos divinos", 12 ejemplares de Psilocybe cubensis, en una ceremonia nocturna, llevada según las costumbres y ritos de los naturales de la región. Los efectos experimentados concuerdan en todo con el cuadro típico de síntomas antes expresados. Al otro día de la ingestión, el autor no presentó ningún estado anormal, a no ser 
un zumbido de los tímpanos, que persistió durante las cuarenta y ocho horas subsiguientes a la ingestión.

La psilocybina se ha experimentado sobre enfermos mentales en la Facultad de Medicina de París (J. Delay y colaboradores, 1958, según Heim v Wasson, 21) probando su acción psico-fisiológica con resultados muy halagadores.

\section{Cultivos de los hongos alucinógenos}

Los hongos alucinógenos del género Psilocybe son susceptibles de culvarse en medios artificiales o naturales, en condiciones de laboratorio, según lo han podido demostrar las magníficas investigaciones de Hiem en colaboración con Cailleux en París $(10,13,21)$, así como Singer y colaboradores en EE.UU. (30,32) y M. Zenteno y T. Herrera en el Instituto de Biología de la UNAM (40). Las técnicas empleadas no son más que una modificación de las que se siguen en el cultivo de los hongos comestibles, tales como Agaricus bisporus (Lange) Singer (=A. Hortensis (Cooke) Konr. et Maubl.).

El autor ha logrado desarrollar sobre agar con extracto de malta, micelio de cuatro de las principales especies de Psilocybe de la sección Caerulescentes, manteniendo asi, 38 cepas de Ps. mexicana, Ps. cubensis, Ps duplicados que Singer amablemente le obsequió, así como de las que él personalmente sembró en Huautla de Jiménez, Oax.

Todas las investigaciones concuerdan en que Ps. cubensis es la especie que más se adapta a las condiciones del laboratorio, a una temperatura de 21 a $25^{\circ} \mathrm{C}$, en medio ácido ( $\mathrm{pH} 5.5$ ) y en condiciones de iluminación natural normal por ser fototrópica positiva, como la mayoría de las especies de Psilocybe, excepto Ps. candidipes que parece ser negativo.

\section{Investigaciones ecológicas}

La ecología micológica, ha sido una de las ramas menos estudiadas, de este modo las investigaciores hasta ahora realizadas sobre los hongos alucinógenos mexicanos, han tenido carácter taxonómico, químico, fisio'ógico, etnológico, etc., pero no ecológico.

Desde 1956, el autor ha estado enfocando sus estudios hacia los hongos alucinógenos mexicanos y en especial a su ecología, tratando de esclarecer el número de especies, sus habitats, su distribución en el país y los factores que la motivan, a modo de delimitar en el mapa las áreas de distribución (Guzmán, 6).

El estudio del habitat ha venido comprendiendo el anális:s biológico, físico y químico de los tipos de sustratos en los que prosperan las diferentes especies. Se ha encontrado que las espec:es alucinógenas se desarrollan sobre sustratos tales como la madera podrida (lignícolas), estiércol (f'mí- 
colas), húmus y restos vegetales diversos (humícolas) y tierra (terrícolas). En este último caso ( la textura varía desde arcillosa hasta casi arenosa, con abundante vegetación herbácea o totalmente desprovista de ella.

Especies lignicolas son Psilocybe yungensis, Ps. isaurii, Ps. caerulipes var. gastoni y Conocybe siligenoides. Especies fimícolas son Ps. cubensis y todos los Panaeolus y afines; sin embargo, Ps. cubensis se desarrolla a veces sobre estiércol casi reincorporado al suelo o sobre restos vegetales abonados, tal como lo ha observado el autor en Necaxa, Pue. y en Huautla de Jiménez, Oax. Las especies humícolas son las mismas terrícolas, ya que el margen de variabilidad es bastante grande entre ellas, por ejemplo, Psilocybe muliercula se encuentra muchas veces en sustratos formados por conglomerados con textura arenosa, en las paredes de las barrancas erosionadas, pero también prosperan sobre restos vegetales diversos (hojarasca y restos de madera) (5). Psilocybe caerulescens var. Mazatecorum se desarrolla la mayoría de las veces, sobre arcilla desprovista totalmente de vegetación aunque también llega a hacerlo sobre bagazo de caña de azúcar o en humus de los cafetales (este último caso, según Heim, es probable que se refiera a otra nueva varedad de la espece, var. umbrofila Heim) (21). Ps. Aztecorum prospera habitualmente en el suelo de las praderas subalpinas del Pinetum Hartwegii; algunas veces su desarrollo parece ir ligado a los restos de madera quemada y semipodrida.

La gran mayoría de los hongos psicotrópicos mexicanos son subtropicales, excepto Ps. Aztecorum que es típico de las regiones subalpinas y Ps. muliercula que sólo ha sido citado de la zona de Abietum religiosae del Nevado de Toluca (5). Las dos especies de Cordyceps relacionadas con los hongos alucinógenos. caracterizadas por ser parásitas de varias especies de Elaphomyces, parecen ser exclusivas de los bosques de Pinus y Quecus del país.

La fenología ha puesto de manifiesto que las especies subtropicales se desarrollan en los meses de junio a agosto y las especies de las zonas templadas, de agosto a octubre.

El estudio de las áreas ha sido difícil de desarrollar debido a su extensión y heterogeneidad; factores de tipo geográfico entre otros, han impedido un avance rápido de las investigaciones. No obstante, se pueden senalar sobre el mapa todas las zonas subtropicales con bosque de tipo Deciduo, como sospechosas para el desarrollo de hongos alucinógenos del género Psilocybe sección Caerulescentes, estando así, Huautla de Jiménez, Oax., Mazatlán, Oax. (y otros pueblos mixes), San Agustín Loxicha, Oax., Yaitepec, Oax. Jalapa, Ver. y Huauchinango, Pue. (Necaxa, Pue.), como lugares plenamente confirmados en cuanto a la existencia de tales especies, no así la Sierra de Guatemala, Tamps. Xilitla, S.L.P., Omilteme, Gro. y las zonas subtropicales del Estado de Chiapas, que son solamente sospechosas. 


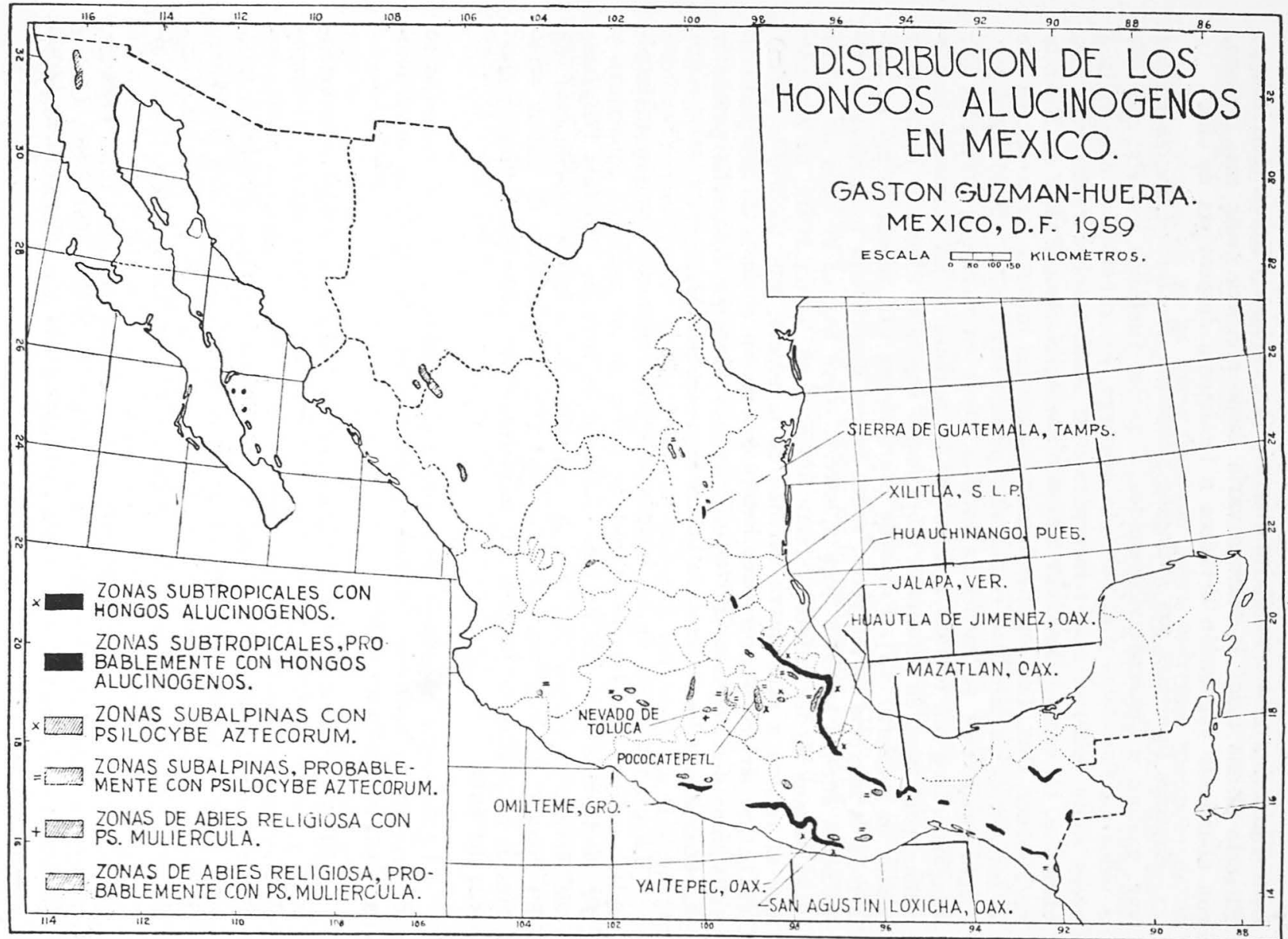


Respecto a la distribución de Psilocybe Aztecorum, se pueden señalar como sospechosas, aquellas zonas subalpinas del país, con exposición E y una altitud variable entre 3000 y 4000 m.s.n.m., con bosque de Pinus Hartwegii, tales como las del Nevado de Colima, Sierra de Michoacán, Nevado de Toluca, Sierra del Ajusco, Pico de Orizaba, Sierra de Puebla, Sierra de Nuevo León, Sierra de Oaxaca y Volcán Tacaná, Chis. Hasta ahora Ps. Aztecorum, sólo ha sido colectado de la región del Paso de Cortés en la Sierra Nevada y en La Malinche, Tlax.

Psilocybe muliercula sólo se ha localizado en el Nevado de Toluca, a una altitud de 3150-3500 m.s.n.m., dentro de la llamada Barranca del Diablo, en el Abietum religiosae (Guzmán, 5); es probable que tal especie exista en otras regiones, con un clima semejante, como lo son las restantes zonas de Abietum religiosae del país.

\section{BIBLIOGRAFIA}

1. Anónimo, 1956. "El valor y un hongo". Revista Visión, Sec. de Medicina, pág. 48. Número de diciembre.

2. Aguirre-Beltrán, G., 1955. "Medicina y Magia. El Proceso de Aculturación y el Curanderismo en México". México, D. F.

3. Barrera, A., 1959. Comunicación personal.

4. Guerra, F. у H. Olivera, 1954. "Las Plantas Fantásticas de México". Imp. Diario Español, México, D. F.

5. Guzmán H., G., 1958. "El Habitat de Psilocybe muliercula Singer. \& Smith (Ps. Wassonii Heim), Agaricaceo alucinógeno mexicano". Rev. Soc. Mex. Hist. Nat. Vol. XIX (1-4) :215-229.

6. ..............., 1959. "Estudio Ecológico de los Hongos Alucinógenos Mexicanos", Tesis Profesional, Escuela Nal. de Ciencias Biológicas. En Prensa.

7. Heim, R., 1956. "Les champignons divinatoires utilisés dans les rites des Indiens Mazateques, recuellis au cours de leur premier voyage au Mexique, en 1953, par Mme Valentina Pavlovna Wasson et M. R. Gorgon Wasson”. Comptes Rendus Acad. Sci. París. 242: 965-968.

8. .............., 1956. "Les champignons divinatoires recueillis par Mme Valentina Pavlovna Wasson et M. R. Wasson au cours de leurs missions de 1954 et 1955 dans les pays mije, mazatéque, zapotéque et nahua du Mexique méridional et central”. Idem, 242:1389-1395.

9. .............., 1957. "Les Agarics hallucinogénes du genæe Psilocybe recueillis au cours de notre récente mission dans le Mexique méridio- 
nal et central en compagnie de M. R. Gordon Wasson”. Idem, 244: 695-700.

10. ..............., y $R$. Cailleux, 1957. "Culture pure et obtention semi-industrielle des Agarics hallucinogenes du Mexique”. Idem, 244:3109-3116.

11. ................, 1957. "Analyse de quelques expériences personelles produites par 1'ingestion des Agarics allucinogenes du Mexique". Idem, $245: 597-603$.

12. ..............., 1957 (1958). "Sur les Psilocybes hallucinatoires des Azteques et sur le microendémisme des Agaries utilisés par les Indiens du Mexique a des fins divinatoires". Idem, 245:1761-1765. y Revue de Mycol. Vol XXII (3) : 300-305.

13. ..............., A. Brack, H. Kobel, A. Hofmann y R. Cailleux, 1958. "Déterminisme de la formation des carpophores et des sclérotes dans la culture du Psilocybe mexicana Heim, Agaric hallucinogene du Mexique, et mise en évidence de la psilocybine et de la psilocine", Idem". 246:1346-1351 y Revue de Mycol. Vol. XXIII (1):106-113.

14. …..........., y A. Hofmann, 1958. "Isolement de la psilocybine a partir" du Stropharia cubensis Earle et d'autres especes de champignon halucinogenes mexicains appartenant au genere Psilocybe”. Idem, 247: $557-561$.

15. ..............., 1957. "Breves latinae diagnoseis hallucinogenarum mexicanarum Psilocybarum ad fera specimina pertinentium". Revue de Mycol. Vol XXII (1):77-79.

16. ..............., 1957. "Diagnose latina Conocybe siligenoides Heim". Idem, Vol. XXII (2):197-198.

17. ............... 1958. "Diagnose latine du Psilocybe Wassonii Heim, espéce hallucigene des Azteques". Idem, Vol. XXIII (1):119-120.

18. ............... y $R$. Cailleux, 1958. "Latina diagnosis Psilocybes sempervi vae Heim et Cailleux, speciei mutuntis hallcinogenae mexicanae perculturam obtentae". Idem, Vol. XXIII (3):352-353.

19. ................, 1958. "Le syndrome narcotien chez les champignons a action cérebrale”. Histoire de la Médecine. No. VIII :1-16.

20. ............., 1958. "Les actions nerveuses provoquées par les champignons". Science et Nature. No. 29:1-8.

21. …........... y R. G. Wasson (con la colaboración de A. Hofmann, R. Cailleux, A. Cerletti, A. Brack. H. Kobel, J. Delay, P. Pichot, Th. Lempériere y P. Nicholas-Charles, 958 (1959). "Les Champignons Hallucinogenes du Mexique”. Editions du Muséum Nat. d’Hist. Naturelle. París. 
22. Hofmann, A., R. Heim, A. Brack y H. Kobel, 1958. "Psilocybin, ein psichotroper Wirkstoff aus mexikanischen Rauschpilz Psilocybe mexicana Heim". Experientia, XIV (3) :107-112.

23. Krieger, L. C. C., 1935. "A popular guide to the higher fungi (mushrooms) of New York State”. N. Y. State Mus. Handb. 11, Albany.

24. Ramsbotton, J., 1954. "Mushrooms and Toadstools. A study of the activities of fungi". Collins, London.

25. Reko, B. P., 1945. "Mitobotánica Zapoteca". México, D. F.

26. Sahagún, Fr. Bernardino de, 1955. "Historia General de las Cosas de la Nueva España”. Ed. Alfa, México, D. F.

27. Schultes, R. E., 1939. "Plantae mexicanae II. The Identification of Teonanacatl, a narcotic Basidiomycete of Aztec". Botan. Leaflets Harv. Univ. 7 (III) :37-54.

28. Singer, R., 1951 (1949). "The Agaricales (mushrooms in modern taxonomy". Lilloa $22: 1-832$.

29.

1958. "Pilze, die Zerebralmyzetismen verusachen”. Bull. Suisse de Mycol. 36 (6):81-89.

30. ..............., 1958. "Mycological Investigat:ons on Teonanácatl, the mexican Hallucinogenic Mushrroom. Part. I. The History of Teonanácatl, Field Work and Culture Work". Mycologia $50(2): 239-261$.

31. .............. y A. Smith, 1958. "Mycological Investigations on Teonanácatl, the mexican Hallucinogenic Mushrrom. Part. II. A Taxonomic Monograph of Psilocybe, section Caerulescentes". Idem. 50(2):262303.

32. (con la colaboración de A. H. Smith, S. I. Stein, y R. W. Ames) 1958. "Observations on Agarica causing cerebral Mycetisms". Mycopathologia et Mycologia applicata, Vol IX (4):261-284.

33. .............. y A. H. Smith, 1958. "New species of Psilocybe". Mycologia, 50 (1):141-142.

34. ….........., A. H. Smith y G. Guzmán H., 1958. A new species of Psathyrella". Lloydia, 21(1):26-28.

35. .............., 1958. "Fungi Mexicani, Series secunda - Agaricales". Sydowia, Vol. XII (1-6):221-243.

36. Villa-Rojas, A., 1957. "Las drcgas alueinantes de los sortilegios mixes: el ololiuqui y el teonanácatl”. Periódico Novedades, Sección de Cultura, 21 de agosto. 
37. Wasson, V. y R. G. Wasson, 1957. "Mushrooms Russia and History". Pantheon Books, N. Y.

38. Wasson, R. G., 1957. "En busca del hongo mágico". Revista Life en español, pág. 38-51. Mes de mayo.

39. ............... 1959. "The Hallucinogenic Mushrooms of Mexico: an adventure in ethnomycological exploration". Transactions of N. Y. Academy of Sciences, Ser. II, Vol. 21 (4) :325-339.

40. Zenteno, M. y T. Herrera, 1958 (1959). Hongos Alucinantes de México. Datos biográficos. Obtención de carpoforos de Psilocybe cubensis (Earle) Sing An. Inst. Biol. Méx. XXIX (1-2) :49-72. 